\title{
Relação entre hipertensão arterial sistêmica e diabetes mellitus tipo 2
}

\author{
André Pozzobon Capeletti ${ }^{1}$; Lilian Fenalti Salla²
}

\section{Resumo}

A prevalência de indivíduos com diabetes mellitus tipo 2 e/ou hipertensão arterial sistêmica vem aumentando. Isoladamente, cada doença determina inúmeros problemas para o paciente; associadas, a situação se agrava ainda mais. A presença de fatores de risco que se relacionam com ambas as doenças, como obesidade, sedentarismo, hipercolesterolemia, hábitos de vida inadequados, entre outros, fazem com que as patologias em questão sejam muito presentes na população. As consequências de um tratamento não precoce e inadequado são diversas e, muitas vezes, determinam a morte do paciente. Entender a relação entre diabetes mellitus tipo 2 e hipertensão arterial sistêmica é de grande relevância para o professional de saúde, já que a presença deles em um mesmo paciente determina uma abordagem mais cuidadosa. O objetivo deste trabalho foi analisar, através de uma revisão bibliográfica, o conteúdo exposto disponível nas revistas indexadas MEDLINE e SciELO sobre essa relação, destacando seus pontos principais.

Palavras Chave: hipertensão arterial sistêmica; diabetes mellitus tipo 2; hábitos de vida

\footnotetext{
${ }^{1}$ Graduando em Medicina pela Universidade Federal de Santa Maria. E-mail: pozzobon.capeletti@hotmail.com.

${ }^{2}$ Graduada em Medicina pela Universidade Federal de Santa Maria (1987), Especialização Residência Médica em Pediatria pela Universidade Federal de Santa Maria (1989), Mestre em Educação pela UFSM (2001), Doutora em Educação em Ciências - Química da vida e saúde (2010). Professora Adjunta do departamento de Morfologia da UFSM. Trabalha com projetos de Promoção da Saúde na Escola pela perspectiva da Alfabetização Científica no Ensino em Ciências.
} 


\section{INTRODUÇÃO}

A hipertensão arterial sistêmica, definida por medida da pressão arterial maior ou igual a 140/90 mmHg, é uma comorbidade comum em pacientes com diabetes mellitus tipo 2. Sua prevalência é cerca de duas vezes maior do que a da população em geral, podendo acometer até $60 \%$ dos pacientes. A hipertensão arterial sistêmica atua sinergicamente com a hiperglicemia, aumentando o risco de desenvolvimento de complicações cardiovasculares, nefropatia diabética e retinopatia diabética (Pinto, 2010). O diabetes mellitus tipo 2 é uma pandemia global e prevê-se que 300 milhões de pessoas o terão em 2025 , em todo o mundo. A prevalência de diabetes mellitus tipo 2 está aumentando rapidamente nos países em desenvolvimento, em particular, tendo um grande impacto na qualidade de vida, na morbidade, na mortalidade e nas despesas de saúde. Um dos maiores fardos do diabetes mellitus tipo 2 é o das complicações crônicas, que surgem com a sua progressão (Hurst, Thinkhamrop e Tran, 2015).

No Brasil, o número de pessoas com diabetes mellitus passou de 4,5 milhões, em 2000, para 11,3 milhões em 2013, com previsão de chegar a 19,2 milhões em 2035, tornando-se o oitavo país do mundo em número de pessoas com diabetes mellitus (Cecílio, 2015). A ocorrência de hipertensão e diabetes mellitus multiplica os fatores de risco para doença micro e macrovascular, resultando em aumento do risco para mortalidade cardiovascular, doença coronariana, insuficiência cardíaca congestiva, doença cerebrovascular e doença vascular periférica. Complicações macrovasculares contribuem para a maioria das mortes em pacientes diabéticos, e a ausência de hipertensão está associada com aumento da sobrevida (Faria, 2002). O diabetes mellitus representa um considerável encargo econômico para o indivíduo e para a sociedade, especialmente quando mal controlado, sendo a maior parte dos custos diretos de seu tratamento relacionado às suas complicações, que comprometem a produtividade, a qualidade de vida e a sobrevida dos indivíduos, e que, muitas vezes, podem ser reduzidas, retardadas ou evitadas (Mclellan, 2007).

A abordagem da hipertensão arterial e do diabetes mellitus é constituída de intervenção medicamentosa e não medicamentosa, sempre acompanhada por mudanças no estilo de vida. Assim, o sucesso do controle das taxas de glicemia e pressão arterial depende da adesão adequada do paciente ao tratamento e de práticas de saúde que estimulem ou facilitem a mudança do estilo de vida (Silva, 2006).

A relação entre hipertensão arterial sistêmica e o diabetes mellitus tipo 2 é visível e muitos pacientes apresentam ambos, interferindo de maneira significativa em suas vidas. O objetivo deste trabalho é fazer uma revisão bibliográfica sobre essa relação, fazendo uso das informações mais relevantes apresentadas nos artigos consultados. 


\section{MÉTODO}

Esta pesquisa é de caráter bibliográfico; foi realizada entre os meses de dezembro de 2015 e fevereiro de 2016. A coleta de informações se procedeu a partir de artigos publicados nas revistas indexadas MEDLINE e SciELO, utilizando na busca dos artigos as palavras "hipertensão arterial" e "diabetes mellitus tipo 2". Por meio de uma revisão da literatura disponível, foi analisada a relação entre a hipertensão arterial sistêmica e o diabetes mellitus tipo 2.

\section{RESULTADOS}

O envelhecimento populacional e as alterações do estilo de vida são apontados como os principais determinantes do acentuado incremento na frequência de diabetes mellitus tipo 2, nos últimos anos. Evidências quanto às alterações no estilo de vida como alimentação não saudável e a falta de atividades físicas regulares, associadas ao aumento da expectativa de vida dos brasileiros têm sido apontados como responsáveis pela prevalência crescente do diabetes mellitus tipo 2 no país (Carolino, 2008).

A frequência de fatores de risco, como hipertensão arterial sistêmica, hipercolesterolemia, sedentarismo, obesidade, entre outros, nos diabéticos tipo 2 é alta e é responsável por uma percentagem significativa de casos com doença cardiovascular presente no momento do diagnóstico. Portanto, as intervenções para evitar diabetes mellitus tipo 2, para serem eficazes, têm de afetar precocemente toda a gama de fatores de risco que estão associados com essa doença e que determinam sua morbidade e mortalidade (Valdés Ramos, 2014). Ademais, as mulheres, em relação aos homens, costumam cuidar mais de si e, historicamente, são responsáveis pelo cuidado com a saúde de suas famílias. Portanto, elas estão mais atentas à sintomatologia das doenças e, geralmente, procuram ajuda precocemente (Carolino, 2008).

Segundo Mclellan:

A intima relação entre o diabetes mellitus tipo 2 e as doenças cardiovasculares leva à hipótese do "solo comum", ou seja, as duas apresentam mesmo componente genético e mesmos antecedentes ambientais, sendo a resistência insulínica considerada um dos principais possíveis antecedentes; o estilo de vida está diretamente relacionado com a incidência de diabetes mellitus tipo 2 e da síndrome metabólica, e a obesidade e o sedentarismo aumentam dramaticamente esse risco. Alguns estudos mostraram que pessoas que consomem uma dieta rica em cereais integrais e ácidos graxos poli-insaturados, associada ao consumo reduzido de ácidos graxos trans e de alimentos com elevado índice glicêmico, apresentam riscos diminuídos para o desenvolvimento de diabetes mellitus (Mclellan, 2007). 
Nos indivíduos com diabetes mellitus, como resultado de alteração da função autonômica e de lesões extensas de órgãos, são frequentes a presença de uma maior variabilidade da pressão arterial, uma marcada resposta ortostática e uma importante deterioração da redução noturna da pressão arterial. Essas características têm implicações diagnósticas, prognósticas e terapêuticas de modo que se recomenda que o número de medidas da pressão arterial para tomada de decisões deve ser maior, a detecção de possível hipotensão para a tomada de decisões também maior, a detecção de hipotensão ortostática deve ser um procedimento de rotina, e a tomada de pressão arterial em ambiente doméstico e, sobretudo, do monitorização ambulatorial da pressão arterial deve realizar-se sempre que possível (Lopez-Jaramillo, 2014).

Nos diabéticos, a hipertensão arterial é duas vezes mais frequente que na população em geral. Pessoas com diabetes mellitus têm maior incidência de doença coronariana, de doença arterial periférica e de doença vascular cerebral. A doença pode também determinar neuropatia, artropatia e disfunção autonômica, inclusive sexual (Silva, 2006). A doença cardiovascular é responsável por substancial morbidade e mortalidade em pessoas com diabetes mellitus tipo 2. Eles têm um risco consideravelmente maior de ter doenças cardiovasculares em comparação com pacientes pareados por idade e sexo sem diabetes mellitus tipo 2 (Herath, Weerarathna e Umesh, 2015). A hipertensão arterial, embora não seja um fator de natureza exclusivamente comportamental, implica consideravelmente no surgimento de complicações microvasculares entre indivíduos com diabetes mellitus, sobretudo quando associada ao longo tempo de diagnóstico e ao não controle glicêmico (Cecílio, 2015).

Ao longo das últimas décadas, várias ferramentas de avaliação de risco foram desenvolvidos para estimar o risco total de doença cardiovascular em indivíduos com diabetes mellitus tipo 2. O escore de risco de Framingham é uma das ferramentas de avaliação de risco mais amplamente utilizadas no mundo todo. Ele baseia-se nos resultados do estudo de Framingham conduzido predominantemente entre a população caucasiana (Herath, Weerarathna e Umesh, 2015). Entre os principais fatores e hábitos que podem auxiliar no controle da doença estão a alimentação adequada e o controle do peso, que podem proporcionar melhoria no controle glicêmico, com consequente redução de riscos para doenças cardiovasculares e melhora da qualidade de vida (Cecílio, 2015).

Conforme Xia:

O diabetes mellitus tipo 2 é uma anormalidade metabólica complexa que aumenta o risco de declínio cognitivo. Cerca de 3/4 dos pacientes com diabetes mellitus tipo 2 vivem com hipertensão. Curiosamente, estudos longitudinais e de autópsia sugerem que a hipertensão é um fator de risco modificável para o desenvolvimento e progressão do declínio cognitivo. Portanto, a hipertensão é um fator de risco provável para o 
comprometimento cognitivo observado em pacientes com diabetes mellitus tipo 2. A relação precisa entre a pressão arterial $e$ função cerebral não foi elucidado em pacientes com diabetes mellitus tipo 2. Pesquisa usando perfusão cerebral, investigada através de tomografia computadorizada por emissão de fóton único, mostra que os pacientes diabéticos apresentam diminuição do fluxo sanguíneo cerebral e que esse está diretamente correlacionado com o desempenho cognitivo (Xia, 2015).

As complicações microvasculares entre os doentes com diabetes mellitus tipo 2 são uma consequência de hiperglicemia prolongada, e estas complicações incluem a neuropatia diabética, nefropatia diabética e retinopatia diabética. A prevalência de complicações microvasculares entre doentes com diabetes mellitus tipo 2 é alta, mas foi demonstrado que variam amplamente entre as populações. Por exemplo, um estudo relatou na China prevalência de 17,8\%,10,7\%,14,8\%, respectivamente, de neuropatia, nefropatia e lesões oculares em pacientes com diabetes mellitus tipo 2. Outro estudo na Austrália relatou a prevalência de $21,9 \%$ de retinopatia diabética em pacientes com diabetes mellitus tipo 2 (Hurst, Thinkhamrop e Tran, 2015).

Segundo Hurst, Thinkhamrop e Tran:

Os pacientes com diabetes mellitus tipo 2 sofrem frequentemente com comorbidades, como hipertensão arterial, obesidade $e$ depressão, e todos foram estabelecidos como comuns nesses pacientes. A comorbidade mais comum associada é a hipertensão. Entender o impacto da comorbidade hipertensão, juntamente com outros fatores de risco, é importante na prevenção de complicações do diabetes mellitus tipo 2. Os pacientes com essa doença e os médicos devem estar cientes da importância do tratamento da pressão arterial, especialmente para pacientes com diabetes mellitus tipo 2 com comorbidade hipertensão (Hurst, Thinkhamrop e Tran, 2015).

Para um controle otimizado da hipertensão na maioria dos pacientes com diabetes mellitus tipo 2, é necessário o uso de duas ou mais drogas. Ademais, em diabéticos tipo 2 com complicações vasculares, a percentagem de pressão arterial não controlada é mais elevada do que naqueles sem estas complicações (Valdés Ramos, 2014). Visto que $30 \%$ dos pacientes normotensos com diabetes mellitus tipo 2 apresentam hipertensão arterial sistêmica mascarada, associada com aumento da excreção urinária de albumina e da espessura das cavidades cardíacas, e que a pressão arterial noturna está associada com a presença da retinopatia diabética, recomenda-se a realização da monitorização ambulatorial da pressão arterial em todos os pacientes com diabetes mellitus, a fim de que seja possível a realização do diagnóstico da hipertensão arterial sistêmica mascarada e avaliação da pressão arterial noturna (Leitão, 2007). A 
monitorização ambulatorial da pressão arterial é um instrumento confiável para monitorar as variações de pressão arterial e tem confiabilidade para uso em pacientes hipertensos com diabetes mellitus tipo 2 (Felício, 2007).

Os pacientes com diabetes mellitus e hipertensão têm um maior risco de doença renal, doença cardíaca coronariana, acidente vascular encefálico e insuficiência cardíaca. A associação com comorbidades como a dislipidemia, estado pró-trombótico e disfunção autonômica contribui para aumentar a morbidade e a mortalidade (LopezJaramillo, 2014). A redução do peso corporal, o controle da hipertensão arterial e da dislipidemia devem ser considerados no tratamento do paciente com diabetes mellitus tipo 2 em unidades básicas de saúde, principalmente adotando-se medidas conservadoras como dieta saudável e atividade física regular. O Sistema Único de Saúde e os profissionais da área devem ser capacitados e estimulados para realizarem as intervenções necessárias para a melhoria da qualidade de vida da população, para promover a redução dos fatores de risco e consequente redução no agravo das doenças crônicas não transmissíveis (Carolino, 2008).

\section{CONCLUSÃO}

Ter conhecimento da comorbidade entre diabetes mellitus tipo 2 e hipertensão arterial sistêmica é de extrema relevância para diminuir a morbimortalidade. Quando as duas doenças estão associadas, o tratamento precoce se faz ainda mais necessário para favorecer um melhor prognóstico. A relação entre hipertensão e diabetes mellitus tipo 2 existe (a primeira geralmente apresenta-se como uma comorbidade da segunda) e suas semelhanças não se dão apenas nos fatores de risco, os quais são iguais em sua maioria (sedentarismo, obesidade, hábitos alimentares inadequados, entre outros).

A tendência atual é o aumento do número de pessoas com hipertensão arterial sistêmica, diabetes mellitus tipo 2 ou ambos, o que constitui uma situação alarmante, já que, por se tratarem de doenças crônicas, os efeitos provocados por um abordagem inadequada ou a ausência de uma abordagem podem ocasionar consequências irreversíveis. A associação dessas patologias com doenças cardiovasculares é bem descrita na literatura e as doenças coronarianas, vasculares cerebrais, arteriais periféricas, entre outras, são fatores de morbidade e mortalidade.

Atuar de maneira contundente e dedicada na busca da identificação correta e precoce do paciente portador de diabetes mellitus tipo 2 e/ou hipertensão arterial sistêmica é dever do professional da saúde. Com um correto diagnóstico, associado a uma terapêutica de qualidade, levando em conta a associação entre essas doenças, um prognóstico positivo é oferecido ao paciente. Há perguntas ainda sem resposta e pesquisas estão em andamento. Indubitavelmente, quanto mais estudos forem feitos na relação entre diabetes mellitus tipo 2 e hipertensão arterial sistêmica, melhor será a compreensão dessas patologias que afetam grande parte população. 


\title{
Relationship between hypertension and type 2 diabetes mellitus
}

\begin{abstract}
The prevalence of individuals with type 2 diabetes mellitus and/or hypertension is increasing. Individually, each disease determines numerous problems for the patient; associated, the situation is even more serious. The presence of risk factors related with both diseases such as obesity, physical inactivity, hypercholesterolemia, inadequate life habits, among others, make the pathologies in question be widespread in the population. The consequences of not premature and inappropriate treatment are diverse and often determine the patient's death. Understand the relationship between type 2 diabetes mellitus and hypertension is of great relevance to the health professional, since their presence in the same patient determines a more careful approach. The aim of this study was to analyze, through a literature review, the exposed content available in journals indexed MEDLINE and SciELO about this relationship, highlighting its main points.
\end{abstract}

Keywords: hypertension; type 2 diabetes mellitus; lifestyle 


\section{Referências}

CAROLINO, Idalina Diair Regla et al. Fatores de risco em pacientes com diabetes mellitus tipo 2. Rev. Latino-Am. Enfermagem, Ribeirão Preto, v. 16, n. 2, p. 238244, Apr. $2008 . \quad$ Disponível em <http://www.scielo.br/scielo.php?script=sci_arttext\&pid=S010411692008000200011\&lng=en\&nrm=iso>. Acesso em 12 Dez. 2015.

CECILIO, Hellen Pollyanna Mantelo et al. Comportamentos e comorbidades associados às complicações microvasculares do diabetes. Acta paul. enferm., São Paulo, v. 28, n. 2, p. 113-119, Apr. 2015 . Disponível em <http://www.scielo.br/scielo.php?script=sci_arttext\&pid=S0103-

21002015000200113\&lng=en\&nrm=iso>. Acesso em 14 Dez. 2015.

FARIA, Alessandra N. et al. Tratamento de Diabetes e Hipertensão no Paciente Obeso. Arq Bras Endocrinol Metab, São Paulo, v. 46, n. 2, p. 137-142, Apr. 2002. Disponível em <http://www.scielo.br/scielo.php?script=sci_arttext\&pid=S000427302002000200004\&lng=en\&nrm=iso>. Acesso em 17 Dez. 2015.

FELICIO, João Soares et al. Reprodutibilidade da medida ambulatorial da pressão arterial em pacientes hipertensos com diabete melito tipo 2. Arq. Bras. Cardiol., São Paulo, v. 88, n. 2, p. 206-211, Feb. 2007. Disponível em $<$ http://www.scielo.br/scielo.php?script=sci_arttext\&pid=S0066-

782X2007000200012\&lng=en\&nrm=iso>. Acesso em 19 Dez. 2015.

HERATH, H. M. M.; WEERARATHA, T. P.; UMESHA, D. Cardiovascular risk assessment in type 2 diabetes mellitus: comparison of the World Health Organization/International Society of Hypertension risk prediction charts versus UK Prospective Diabetes Study risk engine. Vascular Health and Risk Management, 11, 583-589, 2015. Disponível em < http://www.ncbi.nlm.nih.gov/pmc/articles/PMC4654532/>. Acesso em 22 Jan. 2016.

HURST, C.; THINKHAMROP, B.; TRAN, H. T. The Association between Hypertension Comorbidity and Microvascular Complications in Type 2 Diabetes Patients: A Nationwide Cross-Sectional Study in Thailand. Diabetes \& Metabolism Journal, 39(5), 395-404, 2015. Disponível em http://www.ncbi.nlm.nih.gov/pmc/articles/PMC4641969/>. Acesso em 19 Jan. 2016.

MCLELlAN, Kátia Cristina Portero et al. Diabetes mellitus do tipo 2, síndrome metabólica e modificação no estilo de vida. Rev. Nutr., Campinas, v. 20, n. 5, p. 515524, Oct. $2007 . \quad$ Disponível <http://www.scielo.br/scielo.php?script=sci_arttext\&pid=S141552732007000500007\&lng=en\&nrm=iso>. Acesso em 15 Dez. 2015.

LEITAO, Cristiane Bauermann et al. Monitorização ambulatorial da pressão arterial e diabete melito tipo 2. Arq. Bras. Cardiol., São Paulo, v. 89, n. 5, p. 347- 
354, Nov. 2007.

Disponível

em

<http://www.scielo.br/scielo.php?script=sci_arttext\&pid=S0066-

782X2007001700012\&lng=en\&nrm=iso>. Acesso em 19 Dez. 2015.

LOPEZ-JARAMILLO, Patricio et al. Consenso latino-americano de hipertensão em pacientes com diabetes tipo 2 e síndrome metabólica. Arq Bras Endocrinol Metab, São Paulo, v. 58, n. 3, p. 205-225, Apr. 2014. Disponível em $<$ http://www.scielo.br/scielo.php?script=sci_arttext\&pid=S0004-

27302014000300205\&lng=en\&nrm=iso>. Acesso em 17 Dez. 2015.

PINTO, Lana C. et al. Controle inadequado da pressão arterial em pacientes com diabete melito tipo 2. Arq. Bras. Cardiol., São Paulo, v. 94, n. 5, p. 651655, May 2010. Disponível em $<$ http://www.scielo.br/scielo.php?script=sci_arttext\&pid=S0066782X2010000500013\&lng=en\&nrm=iso>. Acesso em 20 Dez. 2015

SILVA, Terezinha Rodrigues et al. Controle de diabetes Mellitus e hipertensão arterial com grupos de intervenção educacional e terapêutica em seguimento ambulatorial de uma Unidade Básica de Saúde. Saúde soc., São Paulo, v. 15, n. 3, p. 180-189, Dec. 2006. Disponível em <http://www.scielo.br/scielo.php?script=sci_arttext\&pid=S010412902006000300015\&lng=en\&nrm=iso>. Acesso em 22 Dez. 2015.

VALDES RAMOS, Eduardo et al. Control de la hipertensión arterial en personas con diabetes tipo 2. Rev Cubana Endocrinol, Ciudad de la Habana, v. 25, n. 2 , agosto 2014.

Disponível em <http://scielo.sld.cu/scielo.php?script=sci_arttext\&pid=S1561$29532014000200005 \& \operatorname{lng}=e s \& n r m=i s o>$. Acesso em 18 Dez. 2015.

VALDES RAMOS, Eduardo; CAMPS ARJONA, María del C; VERDECIA SABORIT, Raúl. Factores de riesgo y enfermedad cardiovascular en diabéticos tipo 2 de diagnóstico reciente. Rev Cubana Invest Bioméd, Ciudad de la Habana, v. 33, n. 2 , jun. 2014. Disponível em $<$ http://scielo.sld.cu/scielo.php?script=sci_arttext\&pid=S086403002014000200009\&lng=es\&nrm=iso>. Acesso em 16 Dez. 2015.

XIA, Wenqing et al. Blood Pressure is Associated With Cerebral Blood Flow Alterations in Patients With T2DM as Revealed by Perfusion Functional MRI. Medicine. 94: e2231， 2015. Disponível em <http://journals.lww.com/mdjournal/Fulltext/2015/12010/Blood_Pressure_is_Associated_With_Cerebral_Blood.28.a spx?trendmd-shared=0> . Acesso em 18 Jan. 2016. 